\title{
Adherence to Screening Colonoscopy: Can We Get Our Recommendations to Stick?
}

\author{
Melinda C. $\operatorname{Rogers}^{1} \cdot$ Rajesh N. Keswani $^{1}$
}

Published online: 19 June 2015

(c) Springer Science+Business Media New York 2015

\begin{abstract}
Although colorectal cancer (CRC) remains the second leading cause of cancer-related mortality in the USA, its incidence and mortality have decreased in men and women since 1997, a reduction attributed, in part, to the increased use of CRC screening modalities [1]. Colonoscopy, regarded by most expert advisory groups as the mainstay of CRC screening, is the only modality that both detects and removes neoplastic polyps from the entire colon. Despite its efficacy, only $20-38 \%$ of the population is adherent to CRC screening guidelines $[2,3]$. Colonoscopy at present is estimated to decrease CRC-related mortality by $53 \%$, with further decrements expected if a greater proportion of individuals received age-appropriate screening [4]. Given limited healthcare resources, information that enables practitioners to focus interventions on populations most likely to be non-adherent to screening colonoscopy is valuable.
\end{abstract}

Multiple patient factors influence adherence to CRC screening [5, 6]. Demographic factors associated with lower rates of screening include female gender, younger age, low income or educational attainment, recent immigrant status, lack of health insurance, and persons of African-American or Hispanic descent [5]. Health factors associated with non-adherence include multiple medical comorbidities and decreased primary care utilization [6]. Nevertheless, little is known about how adherence differs

Rajesh N. Keswani

rkeswani@nm.org

1 Division of Gastroenterology, Northwestern Memorial Hospital, 676 N St Clair, Suite 1400, Chicago, IL 60611, USA among patients who are undergoing surveillance colonoscopy indicated for a history of colorectal polyps or are undergoing screening colonoscopy.

In this issue of Digestive Diseases and Sciences, Greenspan et al. [7] report the results of a prospective study comparing rates of adherence to screening and surveillance colonoscopy in a population of 617 patients seeking care at an urban, tertiary medical center. Of multiple demographic factors studied in patients scheduled to undergo colonoscopy, patients scheduled for screening colonoscopy were significantly less likely to undergo their procedures than those scheduled for surveillance of adenomatous polyps (RR 5.42, CI 2.74-10.75). This intuitive finding represents a new, easily identifiable factor toward which patient adherence efforts can be directed.

While univariate analysis indicated that female gender and the presence of medical comorbidities (represented by clopidogrel use and a history of congestive heart failure) were associated with an increased risk of non-adherence, these were not significant according to multivariable logistic regression analysis, consistent with prior studies [8]. The single factor associated with non-adherence and repeated procedure cancellations according to multivariable analysis, was indication for colonoscopy [7]. Although patients with a prior history of adenomatous polyps are at high risk of the development of adenomas or carcinoma (20-50 \%) at subsequent surveillance colonoscopy, the risk of adenoma or carcinoma in asymptomatic patients undergoing screening colonoscopy can also be as high as $50 \%$ [9]. Thus, it is appropriate to direct efforts toward ensuring adherence to screening colonoscopy in averagerisk screening populations.

The authors also report noteworthy data regarding efforts that may not improve adherence. Prior consultation with a gastroenterologist did not affect colonoscopy 
adherence rates in this study [7], in conflict with prior reports indicating that physician visitation in the preceding year and procedure recommendation by a physician positively influenced rates of adherence to CRC screening [6, 10]. This discrepancy may reflect a higher-risk population with increased rates of comorbid conditions in their study subjects [7]. Furthermore, prior reports of a positive association between physician consultation and recommendation did not differentiate between specialist and primary care interaction, which may additionally account for this variation.

The adherence rates in this study (overall $89.3 \%$ ) are considerably higher than in other published reports $[3,6$, 10]. Reminder phone calls and postcards are effective in increasing rates of CRC screening [11]. In this study population, since all patients received an automated phone call reminder during the study period, it is unknown how this practice affected overall results. Furthermore, the study population included only patients with a scheduled procedure, excluding referred subjects who did not attempt to schedule a colonoscopy, and thus skewing the compliance rates regardless of indication [12].

Although increasing the time between procedure scheduling and anticipated procedure date was associated with multiple procedure cancellations, this was not predictive of overall adherence at 1 year, a discordance that could be explained by a type II error due to inadequate sample size for this measure. Regardless, given the association between procedure cancellations and non-adherence [13], wait time represents a modifiable target at the institutional level in the quest to improve colonoscopy adherence.

Despite effective screening modalities, CRC-related morbidity and mortality continue to be a major public health issue, chiefly due to non-adherence with screening. Greenspan et al. [7] provide evidence to suggest that patients scheduled for screening colonoscopy are at greater risk of non-adherence than those scheduled for surveillance colonoscopy. Further work is required to understand whether this risk factor persists in other populations and, more importantly, what initiatives can be implemented in these at-risk populations to effectively improve overall adherence. The National Colorectal Cancer Roundtable, composed of public, private, and voluntary organizations, recently announced a goal of achieving an $80 \%$ CRC screening rate by 2018 [14]; identifying factors associated with non-adherence, and devising effective interventions, will help achieve this life-saving goal.

\section{References}

1. Edwards BK, Ward E, Kohler BA, et al. Annual report to the nation on the status of cancer, 1975-2006, featuring colorectal cancer trends and impact of interventions (risk factors, screening, and treatment) to reduce future rates. Cancer. 2010;116:544-573.

2. Inadomi JM, Vijan S, Janz NK, et al. Adherence to colorectal cancer screening: a randomized clinical trial of competing strategies. Arch Intern Med. 2012;172:575-582.

3. Swan J, Breen N, Graubard BI, et al. Data and trends in cancer screening in the United States: results from the 2005 National Health Interview Survey. Cancer. 2010;116:4872-4881.

4. Zauber AG, Winawer SJ, O'Brien MJ, et al. Colonoscopic polypectomy and long-term prevention of colorectal-cancer deaths. N Engl J Med. 2012;366:687-696.

5. Steinwachs D, Allen JD, Barlow WE, et al. National Institutes of Health state-of-the-science conference statement: enhancing use and quality of colorectal cancer screening. Ann Intern Med. 2010;152:663-667.

6. Klabunde CN, Cronin KA, Breen N, Waldron WR, Ambs AH, Nadel MR. Trends in colorectal cancer test use among vulnerable populations in the United States. Cancer Epidemiol Biomarkers Prev. 2011;20:1611-1621.

7. Greenspan M, Chehl N, Shawron K, et al. Patient non-adherence and cancellations are higher for screening colonoscopy compared with surveillance colonoscopy. Dig Dis Sci. (Epub ahead of print). doi:10.1007/s10620-015-3664-2.

8. Lukin DJ, Jandorf LH, Dhulkifl RJ, et al. Effect of comorbid conditions on adherence to colorectal cancer screening. J Cancer Educ. 2012;27:269-276.

9. Keswani RN, Yadlapati R, Gleason KM, et al. Physician report cards and implementing standards of practice are both significantly associated with improved screening colonoscopy quality. Am J Gastroenterol. 2015. doi:10.1038/ajg.2015.103.

10. Seeff LC, Nadel MR, Klabunde CN, et al. Patterns and predictors of colorectal cancer test use in the adult U.S. population. Cancer. 2004;100:2093-2103.

11. Hendren S, Winters P, Humiston S, et al. Randomized, controlled trial of a multimodal intervention to improve cancer screening rates in a safety-net primary care practice. J Gen Intern Med. 2014;29:41-49.

12. Denberg TD, Melhado TV, Coombes JM, et al. Predictors of nonadherence to screening colonoscopy. J Gen Intern Med. 2005;20:989-995.

13. Blumenthal DM, Singal G, Mangla SS, Macklin EA, Chung DC. Predicting non-adherence with outpatient colonoscopy using a novel electronic tool that measures prior non-adherence. $J$ Gen Intern Med. 2015;30:724-731.

14. Meester RG, Doubeni CA, Zauber AG, et al. Public health impact of achieving $80 \%$ colorectal cancer screening rates in the United States by 2018. Cancer. 2015. doi:10.1002/cncr.29336. 